\title{
VALIDATION OF A MAGNETO- AND FERRO-HYDRODYNAMIC MODEL FOR NON-ISOTHERMAL FLOWS IN CONJUNCTION WITH NEWTONIAN AND NON-NEWTONIAN FLUIDS
}

\author{
L. Könözsy ${ }^{1}$, P. Scienza ${ }^{1}$, and D. Drikakis ${ }^{2}$ \\ ${ }^{1}$ Centre for Fluid Mechanics and Computational Science \\ Cranfield University, Cranfield, Bedfordshire, MK43 0AL, United Kingdom \\ e-mail: laszlo.konozsy@cranfield.ac.uk, pietro.scienza@icloud.com \\ ${ }^{2}$ Faculty of Engineering \\ University of Strathclyde, Glasgow, G1 1XW, United Kingdom \\ e-mail: dimitris.drikakis@strath.ac.uk
}

Keywords: magnetohydrodynamics, ferro-hydrodynamics, non-isothermal Hartmann flows, Newtonian fluids, non-Newtonian fluids, non-isothermal blood flows.

\begin{abstract}
This work focuses on the validation of a magnetohydrodynamic (MHD) and ferrohydrodynamic (FHD) model for non-isothermal flows in conjunction with Newtonian and nonNewtonian fluids. The importance of this research field is to gain insight into the interaction of non-linear viscous behaviour of blood flow in the presence of MHD and FHD effects, because its biomedical application such as magneto resonance imaging (MRI) is in the centre of research interest. For incompressible flows coupled with MHD and FHD models, the Lorentz force and a Joule heating term appear due to the MHD effects and the magnetization and magnetocaloric terms appear due to the FHD effects in the non-linear momentum and temperature equations, respectively. Tzirtzilakis and Loukopoulos [1] investigated the effects of MHD and FHD for incompressible non-isothermal flows in conjunction with Newtonian fluids in a small rectangular channel. Their model excluded the non-linear viscous behaviour of blood flows considering blood as a Newtonian biofluid. Tzirakis et al. [2] 3] modelled the effects of MHD and FHD for incompressible isothermal flows in a circular duct and through a stenosis in conjunction with both Newtonian and non-Newtonian fluids, although their approach neglects the non-isothermal magnetocaloric FHD effects. Due to the fact that there is a lack of experimental data available for non-isothermal and non-Newtonian blood flows in the presence of MHD and FHD effects, therefore the objective of this study is to establish adequate validation test cases in order to assess the reliability of the implemented non-isothermal and non-Newtonian MHD-FHD models. The non-isothermal Hartmann flow has been chosen as a benchmark physical problem to study velocity and temperature distributions for Newtonian fluids and non-Newtonian blood flows in a planar microfluidic channel. In addition to this, the numerical behaviour of an incompressible and non-isothermal non-Newtonian blood flow has been investigated from computational aspects when a dipole-like rotational magnetic field generated by infinite conducting wires. The numerical results are compared to available computational data taken from literature [2].
\end{abstract}




\section{INTRODUCTION}

For modelling incompressible flows coupled with magnetohydrodynamic (MHD) and ferrohydrodynamic (FHD) approaches, the Lorentz force and a Joule heating term appear due to the MHD effects and the magnetization and magnetocaloric terms appear due to the FHD effects in the non-linear momentum and temperature equations, respectively. This work focuses on the validation of an implemented MHD-FHD model for non-isothermal flows in conjunction with Newtonian and non-Newtonian fluids. The importance of this research work is to investigate the interaction of non-linear viscous behaviour of blood flow with MHD and FHD effects, because its biomedical applications such as magneto resonance imaging (MRI) is in the centre of interest. Due to the fact that there is a lack of experimental data available for non-isothermal and non-Newtonian blood flows in the presence of MHD and FHD field effects, the objective of this study is to establish adequate computational test cases for validation purposes in order to assess the reliability of the non-isothermal and non-Newtonian MHD-FHD models.

A number of theoretical and numerical studies were carried out in the literature to investigate the blood flow behaviour under the action of a magnetic field with particular interest to flows in rectangular ducts and stenosed tubes [1, 2, 3]. Tzirtzilakis et al. [4] proposed a numerical approach to study a blood flow in a three-dimensional rectangular duct where an 8 Tesla magnetic field was generated by an electrically conducing wire on the lower wall of the duct. The blood was considered as a Newtonian electrically non-conducting biofluid and a FHD model was implemented. In their model, the magnetization was assumed linearly dependent on the magnetic field intensity and the magnetic field completely saturated the biofluid. They concluded that two recirculation regions generated slowing down the flow when the magnetic field intensity was increased, and the strong magnetic field also affected the wall stresses near to the area where it was located. In addition to this, a secondary flow was found to be present and strengthen due to the increase of the magnetic field intensity. Loukopoulos and Tzirtzilakis [5] investigated a laminar, viscous incompressible flow of a Newtonian biomagnetic fluid in a two-dimensional channel under the action of spatially varying magnetic field at a low Reynolds number $(R e=$ 250). In this numerical study, the magnetic field was located on the lower wall of the planar channel by taking into account FHD effects with a magnetization linearly dependent on the temperature and magnetic field intensity. They considered realistic blood parameters, different temperatures at the lower and upper walls and a fully developed velocity profile was prescribed as a boundary condition at the inlet. It was observed that a recirculation region developed in proximity of the magnetic field and extended when the magnetic field intensity increased. The flow separated close to the magnetic source and reattached downstream. The velocity profile became fully developed while temperature perturbations were observed at the outlet.

Another numerical study was carried out by Tzirtzilakis and Loukopoulos [1] to investigate the behaviour of blood flows in a two-dimensional small planar channel under the action of a uniform external magnetic field of 8 Tesla generated from two finite disks. The blood was considered as a Newtonian fluid with constant electrical conductivity. The combination of both MHD and FHD models were taken into account in this numerical study and the formation of two recirculation regions was observed where the magnetic field started and ended. In addition to this, two secondary eddies were generated downstream close to the second recirculation region on the lower and upper plates. Due to the rotation of these two vortices, the fluid is pushed on the upper wall within the region of constant magnetic field and the flow slowed down near to the first recirculation region. Furthermore, the velocity profile became fully developed downstream behind the applied uniform magnetic field. It is important to note that the temperature increased 
dramatically closer to the lower plate in proximity of the source of the magnetic field and further increased slowly in the downstream flow regime. In contrast with the velocity field behaviour, the temperature distribution showed a perturbed profile even at the outlet. A variable trend of the wall shear stress inside the magnetized region was also observed in this study.

It has been emphasized in [6] that the effects of temperature field on the numerical solution in conjunction with MHD-FHD models have to be further investigated for biomedical and microfluidic applications. This is due to the fact that there is a lack of experimental data available on the interaction between the temperature and MHD-FHD fields for non-Newtonian blood flows. Therefore this interaction is further investigated and the existing MHD-FHD models are further validated by considering computational aspects in this paper.

Tzirakis et al. [2] conducted a remarkable numerical investigation on the effect of three different magnetic fields for blood flows in four different geometrical configurations. They investigated an incompressible and isothermal flow considering blood as a Newtonian magnetizable and electrically conducting fluid. In their numerical study, a constant magnetic field was applied to plane Couette and Hartmann flows for validating their model implementation. A spatially variable magnetic field generated by an external wire was used for investigating the plane Couette flow and a three-component rotational magnetic field were applied to the flow through a stenosed tube. Their results showed that an irrotational magnetic field does not affect the velocity field, however a rotational magnetic field brakes the flow symmetry inducing separations and altering the velocity distribution. The work of Tzirakis et al. [2] represented an in-depth analysis of magnetic field effects and its investigation of blood flow behaviour, therefore their benchmark test case has also been further validated for non-isothermal flows in this paper.

As a matter of fact, there is a lack of experimental data available for non-isothermal nonNewtonian blood flows in the presence of MHD and FHD effects, therefore the interaction between the temperature field and MHD-FHD models for non-Newtonian blood flows has been investigated in this paper. A two-dimensional, incompressible, laminar and non-isothermal Hartmann flow has been chosen as a benchmark physical problem to study velocity and temperature distributions for Newtonian fluids and non-Newtonian blood flows in a planar microfluidic channel. In addition to this, the numerical behaviour of an incompressible and non-isothermal non-Newtonian blood flow has been investigated from computational aspects when a dipolelike rotational magnetic field generated by infinite conducting wires. The numerical results are compared to available computational data taken from literature [2].

\section{MATHEMATICAL FORMULATION AND SOLUTION METHODOLOGY}

\subsection{Governing equations in conjunction with combined MHD-FHD models}

In this study, for modelling incompressible non-isothermal flows in conjunction with MHD and FHD models, we adopt the mathematical model equations proposed by Tzirtzilakis [7]. The system of governing equations consists of the continuity, momentum and energy equations, thus the mass conservation for incompressible flows can be written in a vector form as

$$
\nabla \cdot \mathbf{u}=0
$$

which is the divergence-free (incompressibility) constraint for the velocity field $\mathbf{u}$. For steadystate incompressible flows, the momentum equation in conjunction with the source terms of MHD and FHD models can be expressed by

$$
\rho(\mathbf{u} \cdot \nabla) \mathbf{u}=\rho \mathbf{g}-\nabla p+\nabla \cdot \underline{\underline{\tau}}+\mu_{0}(\mathbf{M} \cdot \nabla) \mathbf{H}+\mathbf{J} \times \mathbf{B},
$$


where $\rho$ is the fluid density, $\mathrm{g}$ represents the gravity field, $p$ is the hydrodynamic pressure, $\underline{\tau}$ is the viscous stress tensor which can be defined for both Newtonian and non-Newtonian fluids, $\mu_{0}$ is the magnetic permeability of vacuum, $\mathbf{M}$ is the magnetization vector, $\mathbf{H}$ is the magnetic field intensity, $\mathbf{J}$ is the current density vector and $\mathbf{B}$ is the magnetic flux density, respectively. For modelling non-isothermal flows of Newtonian and non-Newtonian fluids with various rheological behaviours, the energy equation for the temperature field can be written as

$$
\rho c_{p}(\mathbf{u} \cdot \nabla) T=\lambda \nabla^{2} T-\mu_{0} T \frac{\partial \mathbf{M}}{\partial T}(\mathbf{u} \cdot \nabla) \mathbf{H}+\frac{\mathbf{J}^{2}}{\sigma}+\underline{\underline{\tau}} \cdots(\nabla \otimes \mathbf{u}),
$$

where $c_{p}$ is the specific heat at constant pressure, $T$ is the temperature, and $\sigma$ is the electric conductivity of the fluid. The first term on the right hand side of the temperature equation represents heat transfer through conduction, the second term represents the magnetocaloric effect of FHD, the third term represents the effect of MHD, and the fourth term $\varphi_{D}=\underline{\underline{\tau}} \cdots(\nabla \otimes \mathbf{u})$ is the viscous dissipation function which is the double dot (inner) product of the viscous stress tensor and the velocity gradient tensor. In the set of governing equations of incompressible nonisothermal flows in conjunction with MHD and FHD models, the current density vector can be expressed by the rotation (curl) of the magnetic field intensity vector $\mathbf{H}$ as

$$
\mathbf{J}=\nabla \times \mathbf{H}=\sigma(\mathbf{u} \times \mathbf{B})
$$

where the electric field effect has been neglected in the present case. We assume that the magnetic flux density field $\mathbf{B}$ is divergence-free as

$$
\nabla \cdot \mathbf{B}=0
$$

For modelling MHD-FHD flows for Newtonian and non-Newtonian fluids, the magnetization force term appears as an external force on the right hand side of the non-linear momentum equation (2) due to the effect of FHD as

$$
\mathbf{f}_{\mathbf{M}}=\mathbf{f}_{\mathbf{F H D}}=\mu_{0}(\mathbf{M} \cdot \nabla) \mathbf{H},
$$

and the Lorentz-force appears due to the effect of MHD which can be written as

$$
\mathbf{f}_{\mathbf{L}}=\mathbf{f}_{\mathrm{MHD}}=\mathbf{J} \times \mathbf{B}=\sigma(\mathbf{u} \times \mathbf{B}) \times \mathbf{B} .
$$

When the fluid is directly sensitive to a magnetic field, its behaviour can be described by FHD model equations and by a magnetic state through the magnetization equation $\mathbf{M}=\mathbf{M}(\mathbf{H}, T)$, which is usually dependent on the magnetic field intensity and the temperature often through too complex or very simple non-realistic relations. However, for some weak magnetic conductors such as blood, a simplified equation can be employed as

$$
\mathbf{M}=\chi \mathbf{H},
$$

which expresses the relationship between the magnetization field $M$ and the magnetic field intensity $\mathbf{H}$ through the magnetic susceptibility of the fluid $\chi$, which can be defined by

$$
\mathbf{H}=\frac{1}{\mu_{0}} \frac{1}{(1+\chi)} \mathbf{B}
$$


thus the momentum equation (2) for incompressible, stationary, non-isothermal flows for Newtonian and non-Newtonian fluids can also be written as

$$
\rho(\mathbf{u} \cdot \nabla) \mathbf{u}=-\nabla p+\nabla \cdot \underline{\underline{\tau}}+\alpha(\mathbf{B} \cdot \nabla) \mathbf{B}+\sigma(\mathbf{u} \times \mathbf{B}) \times \mathbf{B},
$$

and the temperature equation (3) becomes

$$
\rho c_{p}(\mathbf{u} \cdot \nabla) T=\lambda \nabla^{2} T+\frac{\mathbf{J}^{2}}{\sigma}+\underline{\underline{\tau}} \cdots(\nabla \otimes \mathbf{u}),
$$

and the parameter $\alpha$ can be defined by

$$
\alpha=\frac{1}{\mu_{0}} \frac{\chi}{(1+\chi)^{2}}
$$

where $\chi$ is the magnetic susceptibility which expresses the material reactivity to an external magnetic field and can change according to the oxygenation of blood in the present case. For oxygenated blood, the magnetic susceptibility can be chosen as $\chi_{o x}=-6.6 \cdot 10^{-7}$ which is related to the diamagnetic behaviour of blood, and $\chi_{\text {deox }}=3.5 \cdot 10^{-6}$ for de-oxygenated blood according to [2] which characterizes a paramagnetic behaviour. In the present numerical study, the magnetic susceptibility of blood has been considered as de-oxygenated.

The magnetization force appears in Eq. (2) due to the FHD effects which can be written as

$$
\mathbf{f}_{\mathbf{M}}=\mathbf{f}_{\mathbf{F H D}}=\alpha(\mathbf{B} \cdot \nabla) \mathbf{B}=f_{M x} \mathbf{e}_{x}+f_{M y} \mathbf{e}_{y},
$$

where the scalar components of the magnetization vector $f_{M}$ can be defined as

$$
\begin{aligned}
& f_{M x}=\alpha\left(B_{x} \frac{\partial B_{x}}{\partial x}+B_{y} \frac{\partial B_{x}}{\partial y}\right), \\
& f_{M y}=\alpha\left(B_{x} \frac{\partial B_{y}}{\partial x}+B_{y} \frac{\partial B_{y}}{\partial y}\right),
\end{aligned}
$$

which terms appear as additional source terms in the scalar momentum equations for $x$ and $y$ spatial directions for two-dimensional flows. The Lorentz-force appears due to the MHD effects in the momentum equation (2) which can be expressed as

$$
\mathbf{f}_{\mathbf{L}}=\mathbf{f}_{\mathrm{MHD}}=\mathbf{J} \times \mathbf{B}=\sigma(\mathbf{u} \times \mathbf{B}) \times \mathbf{B},
$$

where the scalar components of the Lorentz force vector $\mathbf{f}_{\mathrm{L}}$ can be written as

$$
\begin{gathered}
f_{L x}=\sigma\left(-u B_{y}^{2}+v B_{x} B_{y}\right), \\
f_{L y}=\sigma\left(u B_{x} B_{y}-v B_{x}^{2}\right),
\end{gathered}
$$

which terms again appear as additional source terms in the scalar momentum equations for $x$ and $y$ spatial directions. The magnetic field can be considered as a dipole-like rotational field which was also used by Tzirakis et al. [2] as

$$
\mathbf{B}=\mathbf{B}(x, y)=B_{x} \mathbf{e}_{x}+B_{y} \mathbf{e}_{y},
$$


where the scalar components of the magnetic flux density vector B can be defined by

$$
\begin{gathered}
B_{x}=-C\left[\frac{2\left(x-x_{i}\right)^{2}-r^{2}}{r^{6}}\right], \\
B_{y}=-C\left[\frac{2\left(x-x_{i}\right) \cdot\left(y-y_{i}\right)}{r^{6}}\right],
\end{gathered}
$$

where the dipole radius is defined for a two-dimensional problem as

$$
r=\sqrt{\left(x-x_{i}\right)^{2}+\left(y-y_{i}\right)^{2}},
$$

where $x_{i}$ and $y_{i}$ are the spatial coordinates of field application points, and $C$ is an appropriately chosen constant $\left(C=1.72 \cdot 10^{-10} \mathrm{Tm}^{4}\right.$ in the present numerical study).

\subsection{Non-Newtonian Power-Law Fluid (PLF) Model}

The momentum equation (10) is valid for either Newtonian or non-Newtonian fluids depending on the definition of the viscous stress tensor $\underline{\underline{\tau}}$. Blood is known to be a non-Newtonian fluid which exhibits a shear thinning attitude in particuler to those flows when the Reynolds number is low and the viscous term is dominant. In consequence of this, the non-linear relationship between the viscous stress tensor and the rate of strain has to be taken into account in order to model the non-Newtonain fluid flow behaviour. For non-Newtonian fluids, the dynamic viscosity coefficient which is also called as apparent viscosity is a function of the shear rate as

$$
\mu_{a}=\mu_{a}(|\underline{\underline{\gamma}}|)
$$

therefore the relationship between the viscous stress and shear rate tensors is non-linear as

$$
\underline{\underline{\tau}}=\mu_{a}(|\underline{\underline{\gamma}}|) \underline{\underline{\gamma}}
$$

where the shear rate tensor is defined as two times of the rate of strain (deformation) tensor as

$$
\underline{\underline{\gamma}}=2 \underline{\underline{S}}=\nabla \otimes \mathbf{u}+(\nabla \otimes \mathbf{u})^{T}
$$

and the magnitude of the shear rate tensor can be defined by

$$
\dot{\gamma}=|\underline{\underline{\gamma}}|=\sqrt{\frac{1}{2} \underline{\underline{\gamma}} \cdot \underline{\underline{\gamma}} .}
$$

For modelling non-Newtonian blood flows, different blood rheological approaches are available in the literature such as Power-Law Fluid (PLF), Quemada and Casson models to make an attempt to describe the realistic rheological behaviour of blood [8]. In the present study, we choose the aforementioned PLF model due to its simplicity to model the non-Newtonian nonlinear viscous behaviour of blood. In the PLF blood rheological model, the shear stress is a function of an $n$-power coefficient of the shear strain rate as

$$
\tau=k_{0} \dot{\gamma}^{n}
$$


where $k_{0}$ and $n$ are appropriately chosen coefficients of the PLF model, and these parameters are suggested to be selected as $k_{0}=14.67 \cdot 10^{-7} \mathrm{~Pa} \cdot s^{n}$ and $n=0.7755$ [8]. Relying on the non-Newtonial PLF model, the viscous stress tensor can be written as

$$
\underline{\underline{\tau}}=k_{0} \dot{\gamma}^{n-1} \underline{\underline{\gamma}}
$$

and the Reynolds number definition for the non-Newtonian viscous model can be written [8] as

$$
R e_{P L F}=\frac{\rho h^{n}}{k_{0} U_{\infty}^{n-2}}
$$

\subsection{The Hartmann Flow}

The Hartmann flow in its initial configuration is a planar flow between two parallel flat plates in the presence of a constant magnetic field $\mathbf{B}=B_{0} \mathbf{j}$ normal to the plates. In this case, the Lorentz force component $f_{L x}$ appears in the momentum equation as

$$
f_{L x}=-\sigma u B_{0}^{2}
$$

Due to the fact that the magnetic field is constant and parallel to the y-axis, the flow is affected along the x-direction only, which represents the main advantage of the Hartmann flow for validation purposes. When the Hartmann flow is considered to be a one-dimensional flow, it is possible to derive an analytical solution for the outlet velocity profile which can be compared to the numerical implementation of an MHD model. The outlet velocity profile of a two-dimensional laminar Hartmann flow for a Newtonian fluid can be written [2] as

$$
u(y)=\frac{1}{\sigma B_{0}^{2}}\left(\frac{d p}{d x}\right)\left[1-\frac{\cosh \left(\frac{H y}{h}\right)}{\cosh (H)}\right],
$$

where the dimensionless Hartmann number can be defined as

$$
H_{a}=h B_{0} \sqrt{\frac{\sigma}{\mu}}
$$

The effect of the magnetic field acting on the non-isotermal flow field is studied also in relation to the temperature perturbations. The temperature-velocity coupling is made clear in the energy equation where an additional source term appears as

$$
e_{M H D}=B_{x}^{2} u^{2}+B_{y}^{2} v^{2}
$$

\subsection{Implementation of MHD-FHD model equations}

The combination of MHD and FHD models [2, 7] described in this Section is not available in the ANSYS-FLUENT commercial software package by default. Therefore, the MHD-FHD model for the governing momentum (10) and energy (11) equations in conjunction with the magnetization (13) and Lorentz force (16) source terms has been implemented in the ANSYSFLUENT environment with User-Defined Functions (UDFs) in the C programming language. The scalar components of the magnetization force (14) and (15), and scalar components of the Lorentz force (17) and (18) has been added to the ANSYS-FLUENT Navier-Stokes solver by employing the SIMPLE (Semi-Implicit Method for Pressure-Linked Equations) pressurecorrection algorithm [10] with second-order discretization scheme for the convective flux terms. 
The magnetic field intensity with a single-wire and the rotational magnetic field have been initialized through the UDF implementing its components along the spatial directions. It should be mentioned in terms of the UDF C code implementation that the source terms have to be defined in a "generation-rate/volume" unit. The magnetic field components $\left(x_{i}, y_{i}\right)$ have been stored at the cell-centre on the computational domain and the appropriately chosen $C$ constant has been defined by the actual 4 Tesla field area of action which can be easily adapted and modified according to the requirements. The reason for choosing the magnetic field strength as $4 T e s l a$ is in fact that it was difficult to obtain converged solution for the governing equations (10) and (11) by taking higher values above this value. In order to establish reliable validation test cases for MHD-FHD applications, the convergence of the solution has been considered as a requirement within a small threshold value (smaller than $10^{-7}$ or $10^{-8}$ ). The simulation parameters, geometrical data of a two-dimensional microfluidic benchmark channel including realistic material properties for blood have been summarized in Table 1 .

\begin{tabular}{llll}
\hline Symbol & Value & Description & Units \\
\hline$h$ & 0.01 & Height of the channel & $(\mathrm{m})$ \\
$L$ & 0.12 & Length of the channel & $(\mathrm{m})$ \\
$\mu_{0}$ & $1.256637 \cdot 10^{-6}$ & Magnetic permeability of vacuum & $(\mathrm{H} / \mathrm{m})$ \\
$\chi_{\text {deox }}$ & $3.5 \cdot 10^{-6}$ & Magnetic susceptibility of de-oxygenated blood & $(-)$ \\
$B$ & 4 & Magnetic field intensity & $(\mathrm{T})$ \\
$x_{0}$ & 0.03 & Magnetic field location in direction- $x$ & $(\mathrm{~m})$ \\
$y_{0}$ & $7.42 \cdot 10^{-3}$ & Magnetic field location in direction-y & $(\mathrm{m})$ \\
$C$ & $1.72 \cdot 10^{-10}$ & Rotational magnetic field constant & $\left(\mathrm{T} \cdot \mathrm{m}^{4}\right)$ \\
$\rho$ & 1050 & Density of blood & $\left(\mathrm{kg} / \mathrm{m}^{3}\right)$ \\
$\mu_{N e w t}$ & 0.0035 & Dynamic viscosity of the Newtonian fluid & $(\mathrm{Pa} \cdot \mathrm{s})$ \\
$C_{p}$ & 3617 & Specific heat of blood & $(\mathrm{J} / \mathrm{Kg} \cdot \mathrm{K})$ \\
$k$ & 0.52 & Thermal conductivity of blood & $(\mathrm{W} / \mathrm{m} \cdot \mathrm{K})$ \\
$\sigma$ & 0.8 & Electrical conductivity of blood & $(\mathrm{S} / \mathrm{m})$ \\
$T$ & 300 & Temperature of blood & $(\mathrm{K})$ \\
$T_{l w}$ & 283.65 & Lower wall temperature & $(\mathrm{K})$ \\
$T_{u w}$ & 315.15 & Upper wall temperature & $(\mathrm{K})$ \\
$R e$ & 100 & Reynolds number & $(-)$ \\
$R e_{P L F}$ & 120.025 & Reynolds number of the Power-Law Fluid & $(-)$ \\
$k_{0}$ & $14.67 \cdot 10^{-3}$ & Power-Law viscosity coefficient & $(-)$ \\
$n$ & 0.7755 & Power-Law exponent & $(-)$ \\
$U_{\infty}$ & 0.1 & Inlet flow velocity & $(\mathrm{m} / \mathrm{s})$ \\
\hline
\end{tabular}

Table 1: Simulation parameters, geometrical data and material properties for blood.

For the temperature distribution at the inlet section of the microfluidic channel, a linear temperature profile has been prescribed as a boundary condition as

$$
T_{\text {inlet }}(y)=y\left(T_{u w}-T_{l w}\right)+T_{l w},
$$

and for the velocity field, a uniform inlet velocity distribution has been taken with an average velocity magnitude of $0.1 \mathrm{~m} / \mathrm{s}$ (see Table 1 ). 


\section{RESULTS AND DISCUSSION}

\subsection{Non-isothermal Hartmann flow for Newtonian and non-Newtonian fluids}

In this subsection, we investigate an incompressible, laminar and non-isothermal Hartmann flow in conjunction with Newtonian and non-Newtonian rheological approaches for blood in a two-dimensional microfluidic channel. The computational data has been compared to the numerical data taken from Tzirakis et al. [1] where the fluid has been considered as Newtonian. For this simple preliminary analysis, differently from [1] where a computational mesh consists of 2000 quadrilateral elements was used, a grid of approximately 14000 elements has been found [9] to be more effective in the present study providing high-accuracy and agreement with the results of Tzirakis et al. [1]. A uniform inlet velocity profile has been considered relying on [1] as well as the magnetic field strength has been taken as $B_{0}=25 \sqrt{7}$ Tesla. The Hartmann number defined by Eq. (32) has been taken as equal to 5 . It is important to remark from a practical point-of-view that this value of magnetic field strength is considerably high and unsuitable for biomedical applications. However, the two-dimensional Hartmann flow as a first preliminary case is considered for the validation of the implemented UDFs in the ANSYS-FLUENT environment, because the flow physics is relatively simple and the size of the computational domain is small. The fully developed outlet velocity profile of the Hartmann flow compared to the computational data of Tzirakis et al. [1] is shown in Figure 1.

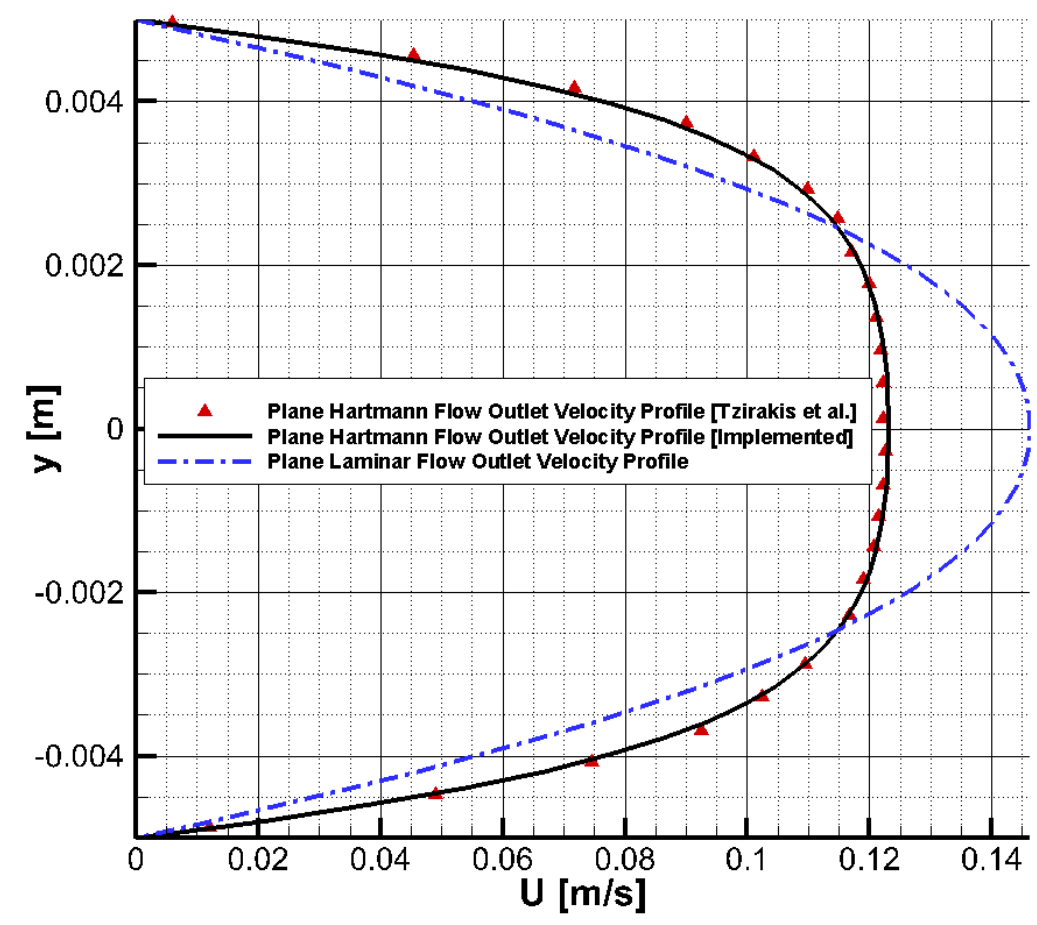

Figure 1: Comparison of outlet velocity profiles for a Hartmann flow in a microfluidic channel against computational data of Tzirakis et al. [2].

It can be seen in Figure 1 that the outlet velocity profile of a fully developed Hartmann flow is different from the fully developed velocity distribution of an incompressible laminar flow 
without the presence of a magnetic field as it is expected. The effect of the magnetic force can be observed as the flow slows down under the action of a constant magnetic field. In addition to this, comparing the Hartmann flow and the laminar flow between parallel flat plates, it can be inferred that the first one develops quicker with a flatter velocity profile at the outlet compared to the second one (see the contour plots in Figures 2 and 3 ). This behaviour is caused by the magnetic field which slows down the blood flow (see Figures 3 and 5). The contour plot of the temperature field in the presence of a constant magnetic field is shown in Figures 4 and 6 .

U-Velocity $[\mathrm{m} / \mathrm{s}] \quad \begin{array}{llllllllllllll}0.01 & 0.02 & 0.03 & 0.04 & 0.05 & 0.06 & 0.07 & 0.08 & 0.09 & 0.1 & 0.11 & 0.12 & 0.13 & 0.14\end{array}$

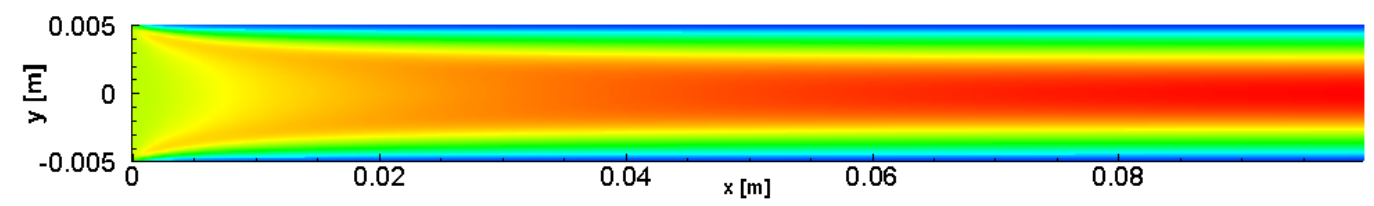

Figure 2: Contours of the velocity component $U$ for a non-isothermal Hartmann flow in a two-dimensional microfluidic channel in conjunction with a Newtonian fluid with a uniform inlet velocity distribution.


Figure 3: Contours of the velocity component $U$ for a non-isothermal Hartmann flow in a two-dimensional microfluidic channel in conjunction with a non-Newtonian fluid (blood) with a uniform inlet velocity distribution.

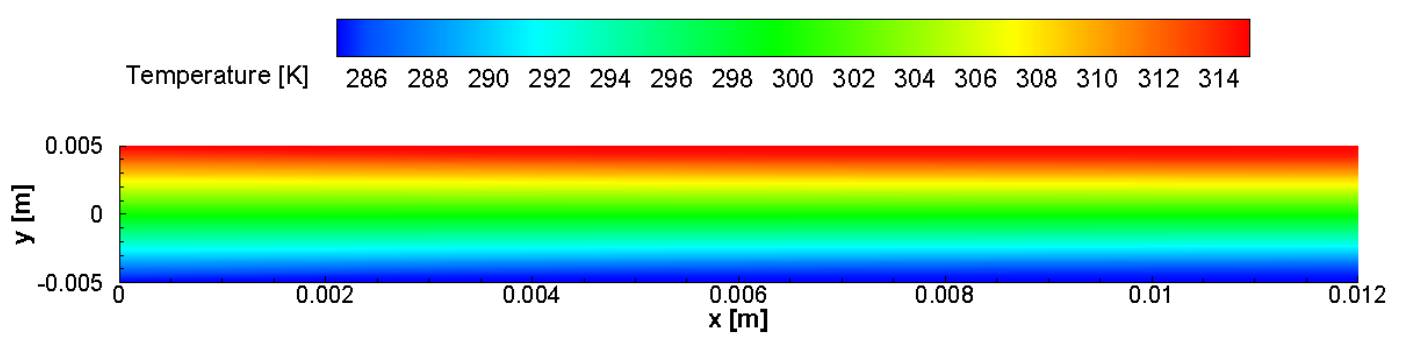

Figure 4: Contours of the temperature field for a non-isothermal Hartmann flow in a two-dimensional microfluidic channel in conjunction with a non-Newtonian fluid (blood) with a linear inlet temperature distribution.

The velocity profiles in a two-dimensional microfluidic channel at different cross-sections have been shown in Figure 5. It can be seen that there are minor differences between the crosssectional velocity profiles for considering blood as a Newtonian and a non-Newtonian fluid 
corresponding to the assumtion that blood can be considered as a weak non-Newtonian fluid. The effect of a constant magnetic field on the incompressible and laminar flow can also be observed in Figures 2 and 3 , because the cross-sectional velocity profiles appear in a roundlike shape compared to the fully developed laminar velocity profile without the presence of a constant magnetic field (see the blue curve in Figure 1).
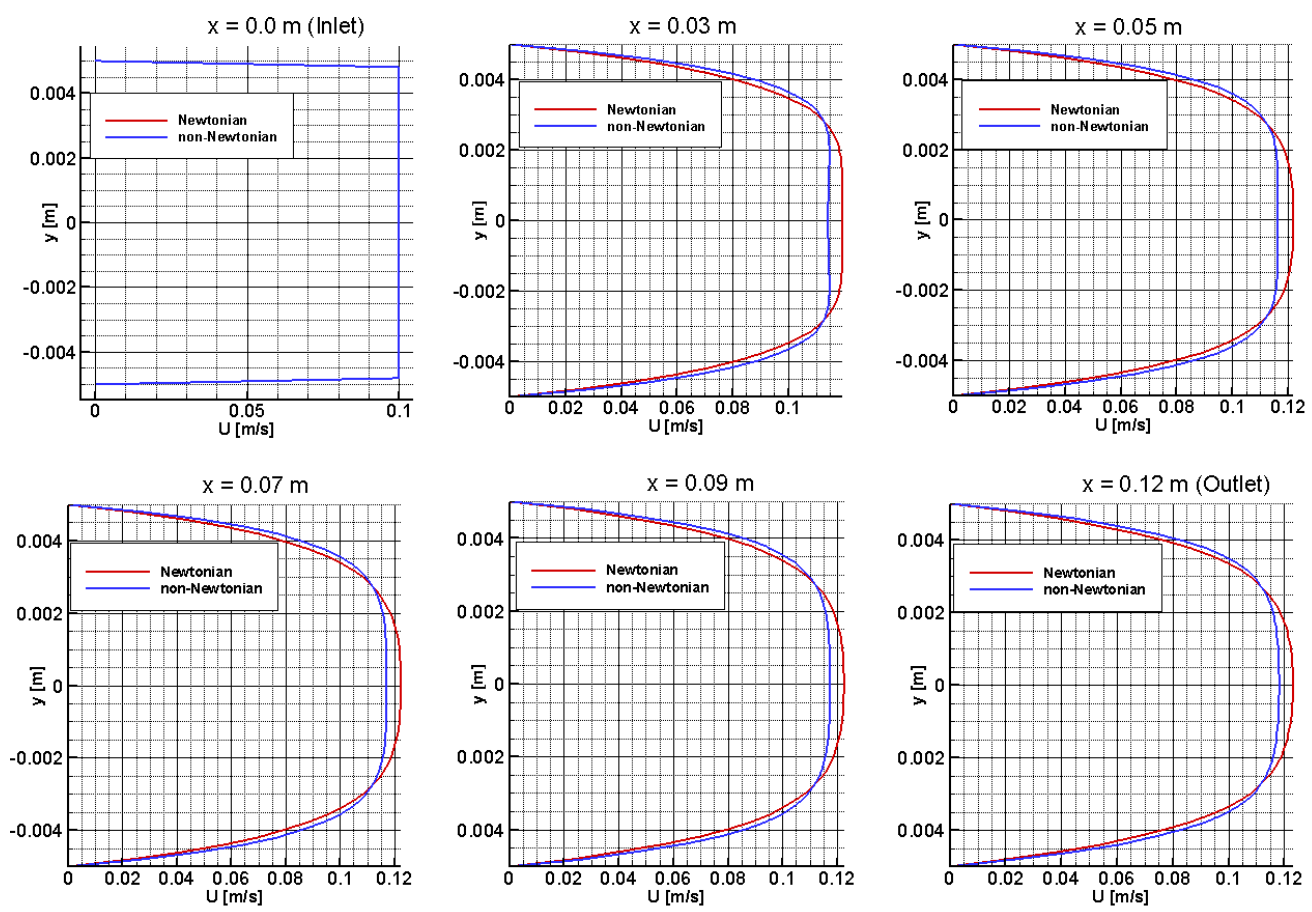

Figure 5: Comparison of velocity profiles for Newtonian and non-Newtonian blood rheological approaches in conjunction with a non-isothermal Hartmann flow in a microfluidic channel at different cross-sections.

For non-isothermal Hartmann flows in conjunction with Newtonian and non-Newtonian rheological models for blood, the temperature field is affected by the presence of the Lorentz force due to the effect of MHD. In this case, the source terms appearing due to the theory of FHD are neglected in the momentum and energy equations. The upper and lower wall temperatures can be found in Table 11, which are $T_{l w}=283.65 \mathrm{~K}$ and $T_{u w}=315.15 \mathrm{~K}$, respectively. The total temperature difference between the lower and upper walls is $31.5 \mathrm{~K}$ which is a relatively small variation, however considering the small size of the microchannel, temperature profiles can also be affected by the presence of a constant magnetic field. It is valid in particular for those problems when a high magnetic field strength is applied perpendicular to the microfluidic channel. However, for the present Hartmann flow case, the effect of the MHD source term on the right hand side of the temperature equation is small due to the fact that the applied magnetic field strength is also small. The inlet temperature profile has been considered as a linear temperature distribution which can be seen in Figure 6. Since the temperature difference between the lower and upper walls as well as the applied constant magnetic field strength is also small, the temperature distribution at different cross-sections of the microfluidic channel exhibits minor differences between Newtonian and non-Newtonian rheological approaches for blood. The presence of the applied constant magnetic field affects slightly the temperature distribution compared to the assumed inlet linear profile. One can see in the following subsections 
that the effect of the applied magnetic field becomes significant when a single wire or two wires are located at different axial coordinates in the microfluidic channel. This fact was also observed by Tzirtzilakis et al. [1] when a strong uniform magnetic field was applied.
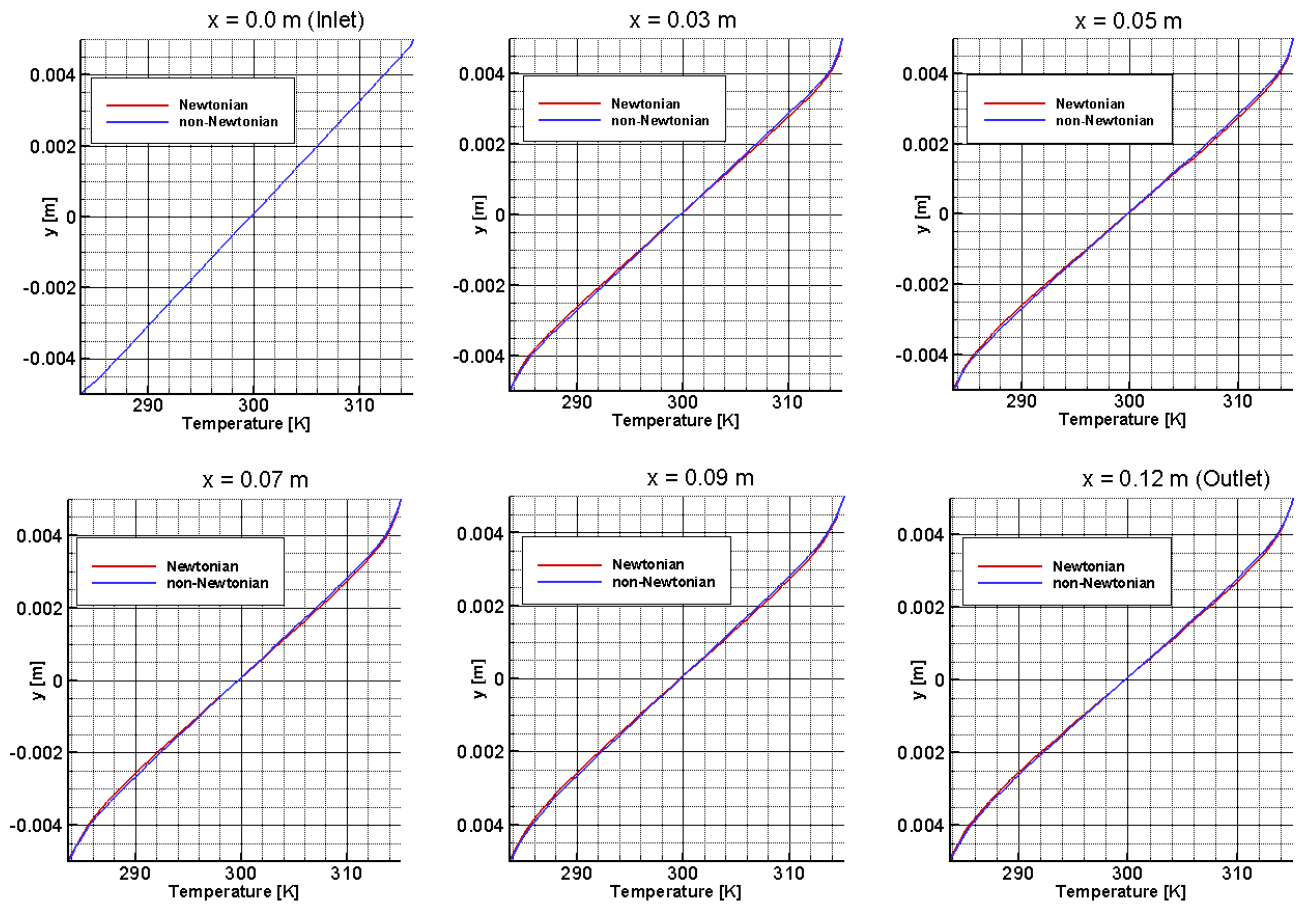

Figure 6: Comparison of temperature profiles for Newtonian and non-Newtonian blood rheological approaches in conjunction with a non-isothermal Hartmann flow in a microfluidic channel at different cross-sections.

The convergence history of the numerical solution for non-isothermal Hartmann flow in conjunction with Newtonian and non-Newtonian blood rheological models can be seen in Figure 7. For solving a steady-state fluid flow problem, the convergence criterion has been prescribed within a small threshold value (smaller than $10^{-8}$ ). The maximum number of iterations for Newtonian and non-Newtonian fluids was 145 to obtain convergence to the approximate solution of the governing equations. The numerical results show that the ANSYS-FLUENT environment by using UDFs in C programming language is capable of solving MHD non-isothermal Newtonian and non-Newtonian fluid flows within a relatively small number of iterations on a fine computational mesh consists of approximately 14000 control volume elements. It has been observed that the magnetic field strength has to be also small in particular to non-Newtonian fluids, otherwise the approximate solution of the system of equations can lead to oscillatory and non-physical solutions. For taking a high value of the applied constant magnetic field strength, the source terms in the momentum (10) and temperature equations (11) might become very stiff, thus a careful numerical treatment and even advanced method development could be required within the framework of an in-house code implementation to obtain physically reasonable solutions for the system of governing equations. The stiffness of the MHD source terms in the set of model equations could lead to a numerical solution when the convergence of the numerical procedure could be difficult to ensure. Therefore, the applied magnetic field strength is not higher than 4 Tesla in the following subsections for the investigated validation test cases to ensure numerical solution which converges to a small threshold value. 

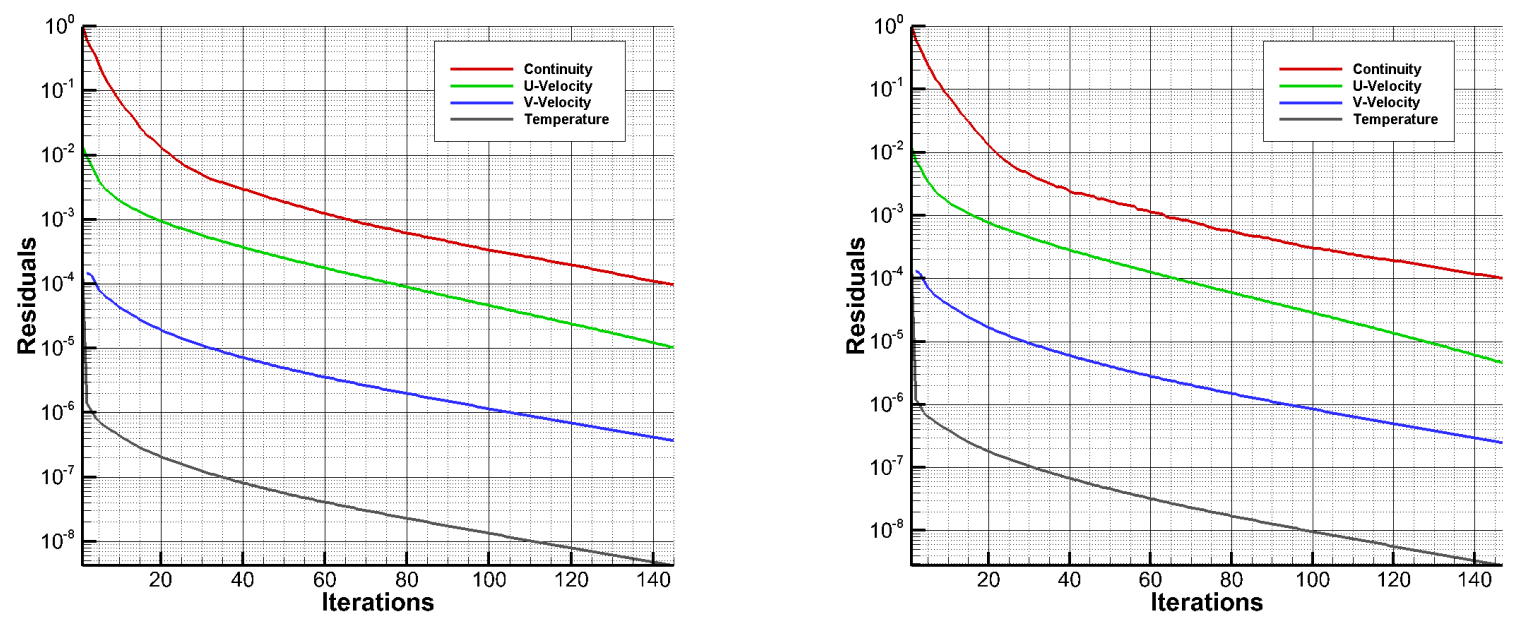

Figure 7: Residual history of non-isothermal Hartmann flow simulations: Newtonian fluid flow (left) and nonNewtonian blood flow (right).

\subsection{Single wire problem}

In this subsection, we present a benchmark problem for solving incompressible, laminar and non-isothermal flows in conjunction with MHD and FHD models and Newtonian and nonNewtonian blood rheological approaches. A single wire has been located in a two-dimensional microfluidic channel at the spatial coordinate $x_{1}=0.03 \mathrm{~m}$ where the magnetic field strength has been taken as 4 Tesla. The local arrangement of the single wire in the two-dimensional microfluidic channel has been shown in Figure 8.

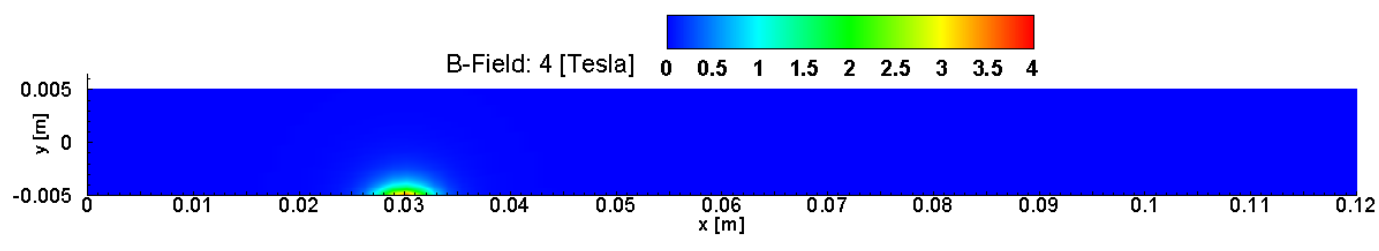

Figure 8: Contours of a single wire induced magnetic field of 4 Tesla on the lower wall at $x_{1}=0.03 \mathrm{~m}$.

This benchmark problem is a hybridization of the single wire model used by Tzirakis et al. [2] and the mathematical model for blood flows and magnetic fields proposed by Tzitzilakis [7]. The magnetization force due to the effect of FHD and the Lorentz force due to the effect of MHD have been taken into account in the momentum equation (10). We assume that the magnetic state through the magnetization equation $\mathbf{M}=\mathbf{M}(\mathbf{H})$ depends only on the magnetic field intensity $\mathbf{H}$, however the magnetization depends on the temperature $T$ in reality. Relying on this assumption which can be used for weak magnetic conductors such as blood [2], the effects of MHD and FHD is taken into account in the momentum equation (10), however, only the effect of MHD appears in the temperature equation (11). This is due to the fact that the temperature derivative of the magnetization vector $\mathbf{M}$ vanishes from the energy equation (3), because it is assumed that the magnetization is function of the magnetic field intensity, but not 
function of the temperature. In other words, it also means that the magneticaloric effect of FHD has been neglected in the MHD-FHD set of model equations in conjunction with Newtonian and non-Newtonian fluids, because the blood can be considered as a weak magnetic conductor.

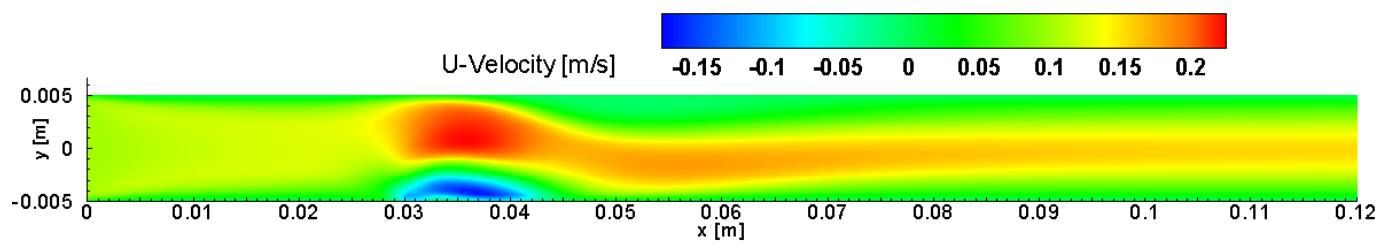

Figure 9: Contours of the velocity component $U$ for a non-isothermal non-Newtonian blood flow in a microfluidic channel with a uniform inlet velocity distribution when the applied magnetic field is induced by a single wire.

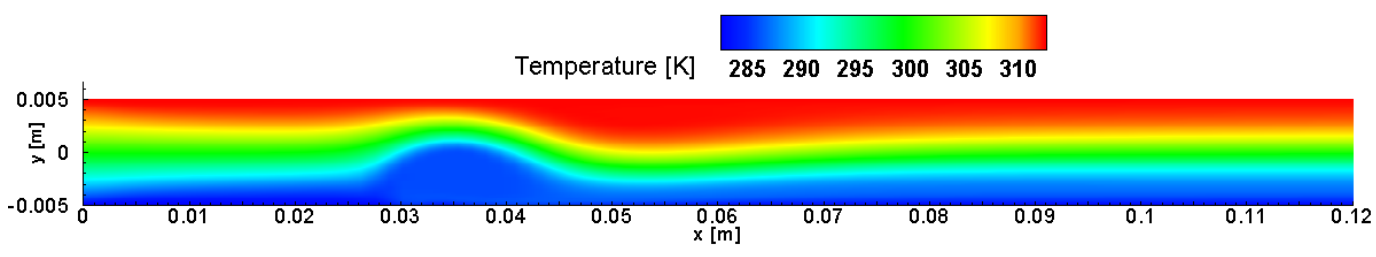

Figure 10: Contours of the temperature field for a non-isothermal non-Newtonian blood flow in a microfluidic channel with a linear inlet temperature distribution when the applied magnetic field is induced by a single wire.

The reason for investigating incompressible and non-isothermal MHD-FHD Newtonian and non-Newtonian fluid flows with the hybridization of Tzirakis et al. [2] and Tzitzilakis [7] MHDFHD models is to study further the interaction between the temperature and MHD-FHD fields for non-Newtonian blood flows, because there is a lack of experimental data available in the literature. In addition to this, there is a lack of well-established and reliable numerical benchmark test case available for verification and validation purposes, thus we also focus on the computational fluid dynamics (CFD) aspects of this physical problem. For steady-state flows, the governing equations (10)-(11) with MHD and FHD models in conjunction with Newtonian and non-Newtonian fluids can be implemented in an in-house code or in a commercial software environment by using User-Defined Functions. The governing equations of the combination of MHD and FHD models [2, 7] described in Section 2 is not available in the ANSYS-FLUENT software package by default, therefore the implementation of a complex multiphysics problem related to MHD and FHD models gives an opportunity to study the strength and limitations of these models from computational aspects. Therefore, it is important to mention from a numerical modelling point-of-view that it has been observed that the presence of the magnetocaloric term of FHD on the right hand side of the temperature equation (3) could lead to numerically unstable and non-physical results when the magnetization vector $M$ is depending on the temperature. This can be explained by the fact that the temperature dependence of the magnetization can be expressed through either too complex mathematical relationships or may be described with very simple and non-realistic functions. As an example, our numerical experience shows that the fully coupled MHD-FHD model for non-isothermal Newtonian flows under the action of a uniform localized magnetic field proposed by Tzirtzilakis and Loukopoulos [1] led to unstable numerical solution by using the ANSYS-FLUENT environment at high dimensionless 
magnetic numbers. In other words, the computational benchmark problem of Tzirtzilakis and Loukopoulos [1] might be difficult to investigate and further validate with a commercial software package even though their research work contains a relevant study on the the interaction between the temperature and MHD-FHD fields by considering blood as a Newtonian fluid. As it has been mentioned above that there is a lack of experimental data available on investigation of the interaction between the temperature and MHD-FHD fields for non-Newtonian blood flows, the computational benchmark presented in this subsection could be used in both in-house and commercial software package environment to validate the implementation of an MHD-FHD model for non-isothermal non-Newtonian blood flows.
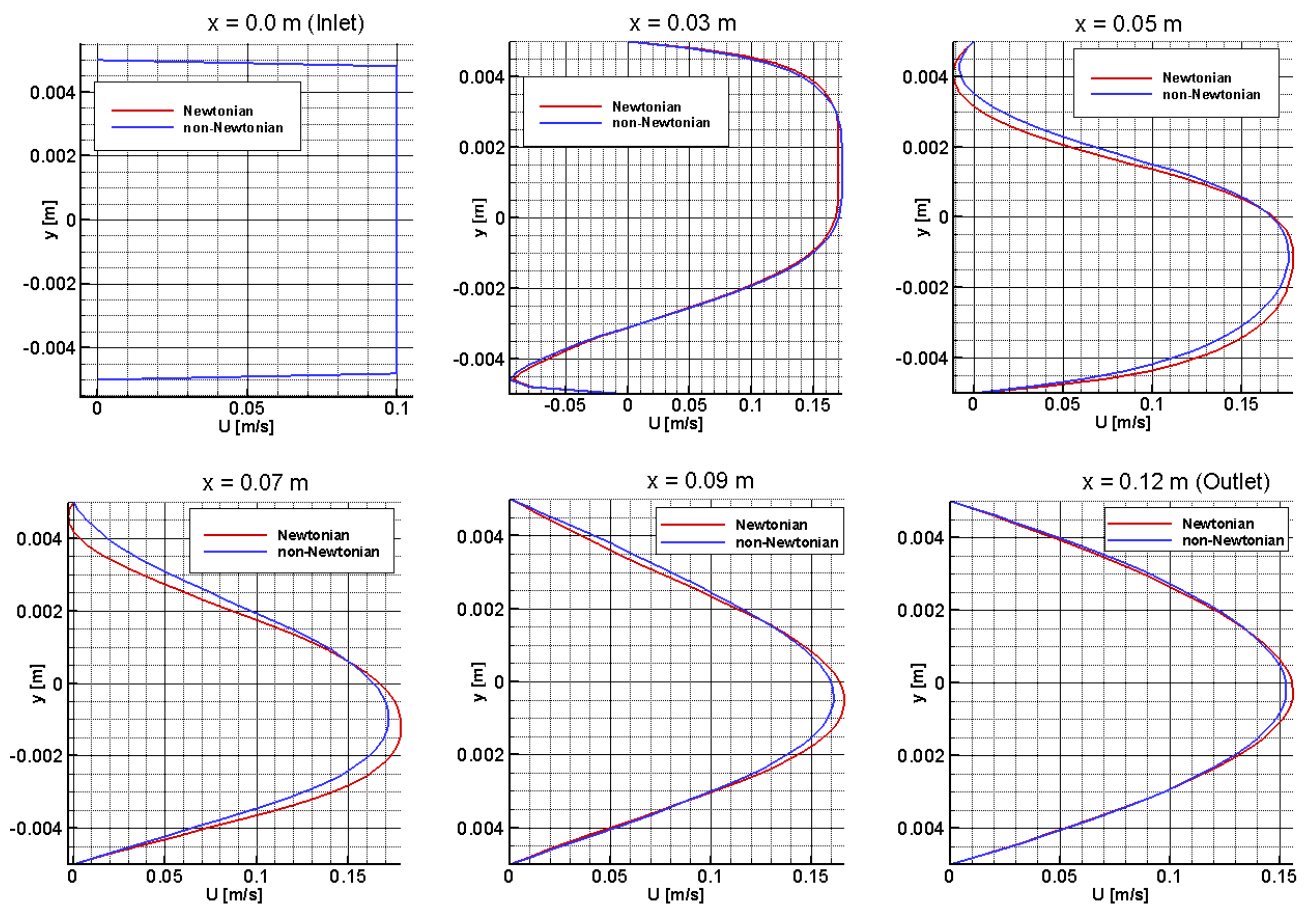

Figure 11: Comparison of velocity profiles for a non-isothermal blood flow in a microfluidic channel at different cross-sections when the applied magnetic field is induced by a single wire.

In the present benchmark problem, the magnetic field is applied through a single wire located in a two-dimensional microfluidic channel and when the temperature dependence of the magnetization is neglected, the effect of MHD and FHD appears in the momentum equation (10) and the temperature equation (11) is only affected by the source term due to MHD. In order to avoid observed numerical oscillations and possible non-physical solution for the benchmark problem presented in this subsection, the MHD-FHD model equations have been set up to cancel out the magnetocaloric effect of FHD on the right hand side of the energy equation (3) which would be a numerically sensitive term in the temperature equation if the magnetization would depend on the temperature field as well. It can be seen in Figure 13 that when the incompressible and non-isothermal flow was influenced by an applied single wire magnetic field along with the temperature independent magnetization, the numerical convergence of the approximate solution of the fully coupled governing equations was ensured for both Newtonian and non-Newtonian blood rheological approaches. In other words, the combination of existing MHD and FHD models [2, 7] proposed in this paper leads to an appropriate computational benchmark test case to 
ensure convergence and accurate solution of MHD-FHD model equations with a convergence criterion smaller than $10^{-7}$ (see Figure 13 up to the magnitude of the applied magnetic field strength of 4 Tesla (see Figures 8 to 12 ).
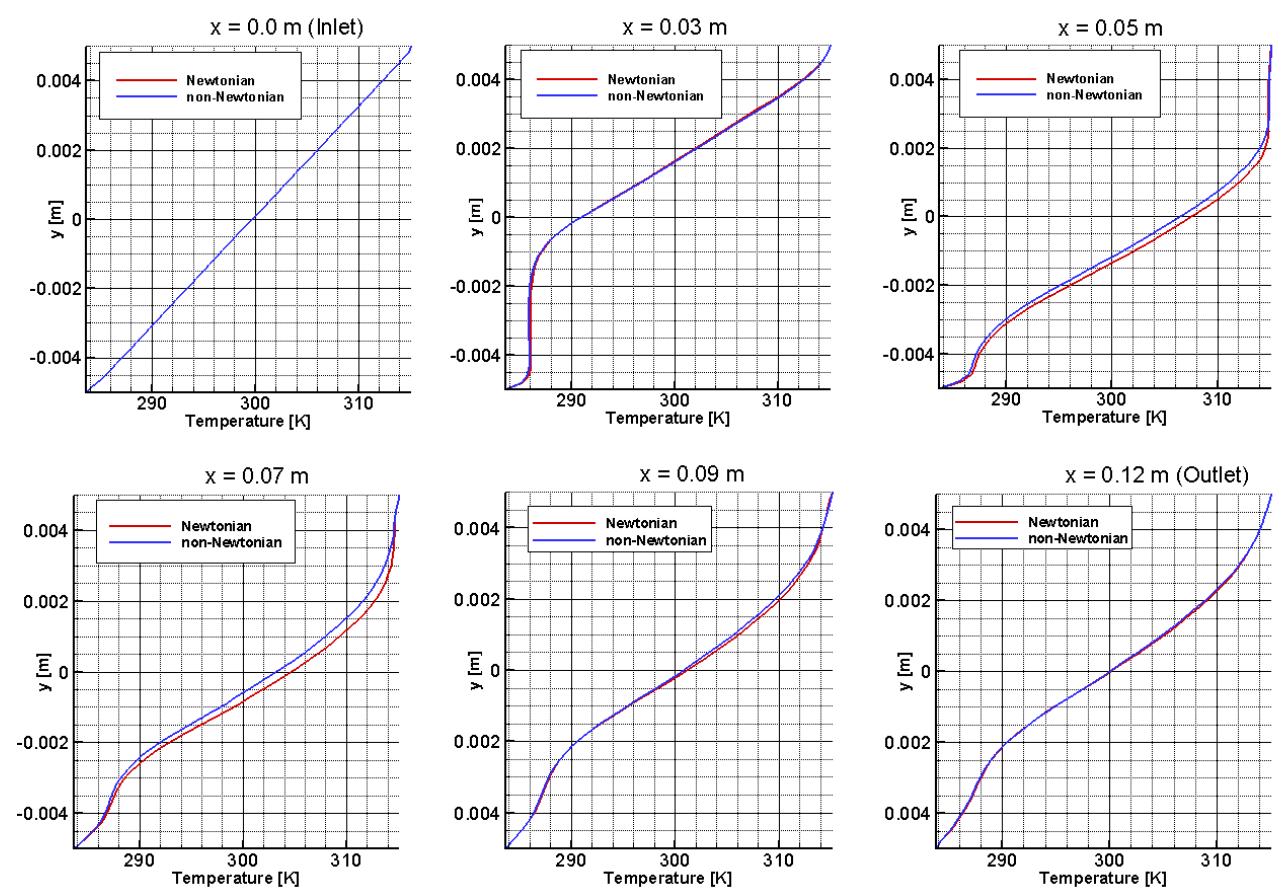

Figure 12: Comparison of temperature profiles for a non-isothermal blood flow in a microfluidic channel at different cross-sections when the applied magnetic field is induced by a single wire.

For incompressible and non-isothermal flow influenced by an applied single wire magnetic field for Newtonian and non-Newtonian fluids, the velocity and temperature profiles at different locations of the two-dimensional microfluidic channel have been shown in Figures 11 and 12 , respectively. The velocity and temperature fields are strongly affected by the location area of the applied magnetic field through a single wire as well as the downstream location behind the wire (see Figures from 9 to 12 ). The velocity field of the incompressible, laminar and non-isothermal flow becomes fully developed far from the applied magnetic field area. It can be seen in Figure 12 that the outlet velocity profile is not affected by the magnetic field strength, thus the profile is similar to the fully developed laminar velocity distribution for both Newtonian and non-Newtonian fluids. The temperature field behaviour is different compared to the non-isothermal Hartmann flow due to the fact that the effects of MHD and FHD terms in the momentum equation $(10)$ has impact on the temperature distribution through convection even if the magnetocaloric effect of the FHD term does not appear in the temperature equation (11). The outlet temperature profile (see Figure 12) exhibits a non-linear behaviour compared to the outlet temperature distribution of the non-isothermal Hartmann flow whereas the profile becomes quasi-linear at the outlet section of the microfluidic channel (see Figure 6). For steady-state Newtonian and non-Newtonian fluid flows, the numerical solution converged to a small threshold value of $10^{-7}$ by performing 450 total number of iterations. For this numerical test case, approximately 3 times more total number of iterations were required compared to the non-isothermal Hartmann flow simulation, because the magnetization force due the effect of FHD has been taken into account on the right hand side of the momentum equation (10). 



Figure 13: Residual history of non-isothermal blood flow simulations for a single wire problem: Newtonian fluid flow (left) and non-Newtonian blood flow (right).

The computational benchmark problem presented in this subsection could be considered as a reliable verification and validation test case for non-isothermal Newtonain and non-Newtonian MHD-FHD fluid flows when microfluidic applications are concerned, because the numerical solution was converged to a very small threshold value of $10^{-7}$ within a relatively small total number of iterations. Furthermore, the obtained results are well-explainable comparing them to the non-isothermal Hartmann flow. It is important to mention again the limitation of the current implementation, because it has been observed relying on numerical experience that when the applied magnetic field strength is higher than 4 Tesla, it was difficult to ensure convergence of the numerical solution for both Newtonian and non-Newtonian fluids. In consequence of this, it is recommended to employ higher than second-order treatment of the convective and/or MHDFHD terms in the system of governing equation in order to overcome the oscillatory numerical behaviour of the stiff MHD and FHD source terms at high dimensionless magnetic numbers.

\subsection{Double wire problem}

In this subsection, a benchmark problem has been presented for an incompressible, laminar and non-isothermal MHD-FHD blood flow in conjunction with Newtonian and non-Newtonian rheological models when the fluid flow is influenced by a double wire arrangement of the applied magnetic field strength. Similar to the numerical example presented in the previous subsection, a wire is located at the spatial coordinate $x_{1}=0.03 \mathrm{~m}$ on the lower wall in a twodimensional microfluidic channel where the magnetic field strength is taken as 4Tesla. In addition to this, another wire is located at the spatial coordinate $x_{2}=0.07 \mathrm{~m}$ on the upper wall where the magnetic field strength is equal to 1.2 Tesla. The set of governing equations are relying on the mathematical model equations proposed by Tzitzilakis [7], however, we define the viscous stress tensor $\underline{\underline{\tau}}$ according to the constitutive equation related to a viscous Newtonian and a power-law non-Newtonian fluid model for non-isothermal blood flows. This benchmark test case represents a more complicated multiphysics problem in conjunction with MHD-FHD flows which triggers off vortical structures in a two-dimensional microfluidic channel similar to the benchmark problem investigated by Tzirtzilakis and Loukopoulos [1] in the presence of 
a localized uniform magnetic field. For this multiphysics problem, we assume again that the magnetic state through the magnetization equation $\mathbf{M}=\mathbf{M}(\mathbf{H})$ depends only on the magnetic field intensity $\mathbf{H}$ and it is independent from the temperature field $T$. It means that the effects of MHD and FHD have been taken into account in the momentum equation (10) and the FHD effect have been neglected in the temperature equation (11). For an incompressible, laminar and non-isothermal non-Newtonian blood flow, the velocity and temperature field contours have been shown in Figures 14 and 15 , respectively.

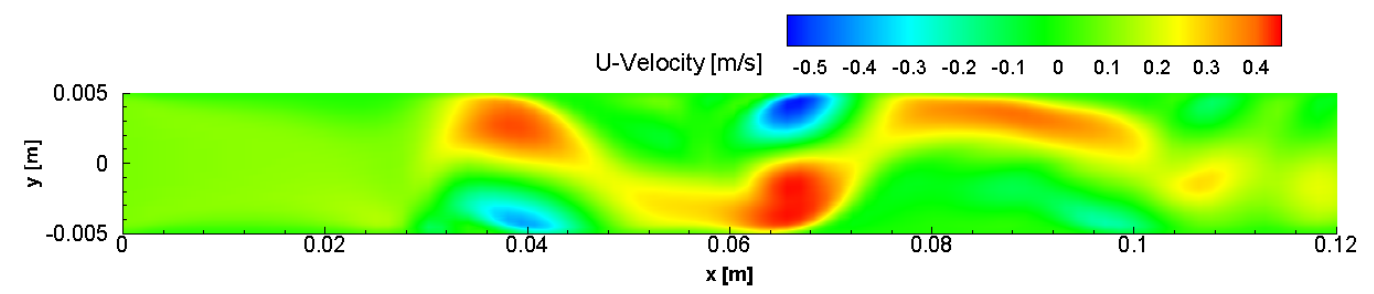

Figure 14: Contours of the velocity component $U$ for a non-isothermal non-Newtonian blood flow in a microfluidic channel with a uniform inlet velocity distribution when the applied magnetic field is induced by a double wire.



Figure 15: Contours of the temperature field for a non-isothermal non-Newtonian blood flow in a microfluidic channel with a linear inlet temperature distribution when the applied magnetic field is induced by a double wire.

Tzirtzilakis and Loukopoulos [1] investigated a non-isothermal Newtonian flow under the action of a uniform localized magnetic field of 8 Tesla which was two times more than the magnitude of the applied magnetic field through a wire for the benchmark problem presented in this subsection. Furthermore, they took into account the magnetocaloric effect of FHD in the energy equation which could be a stiff source term in the set of governing equations. The streamfunction-vorticity formulation was used by Tzirtzilakis and Loukopoulos [1] for solving the MHD-FHD model equations along with a line by line implicit method. Their numerical solution was reduced to solve the discretized system of equations by using the Thomas algorithm which also means that this numerical approach was basically a one-dimensional solution of the discretized governing equations along the axial direction, and the effect of the other direction was taken into account on the right hand side of the system of linear equations. It might be important to highlight that the numerical solution of a fully coupled MHD-FHD governing equations by using an approximate one-dimensional line by line approach is not equivalent to a fully two-dimensional explicit or implicit numerical solution to the set of MHD-FHD model equations. Therefore the convergence properties in conjunction with the presence of stiff 
MHD-FHD source terms could lead to different numerical behaviours when various methods are used for the same computational benchmark problem. According to our numerical experience related to a fully two-dimensional computational approach, the relatively stiff MHD-FHD source terms, implemented in the ANSYS-FLUENT environment, caused numerical instabilities when the magnitude of the applied uniform localized magnetic field was 8 Tesla. In other words, the fully two-dimensional numerical solution of the benchmark problem of Tzirtzilakis and Loukopoulos [1] in the ANSYS-FLUENT environment was not converged to the threshold value of $10^{-5}$ as it was prescribed in [1] for an approximate line by line solution method.
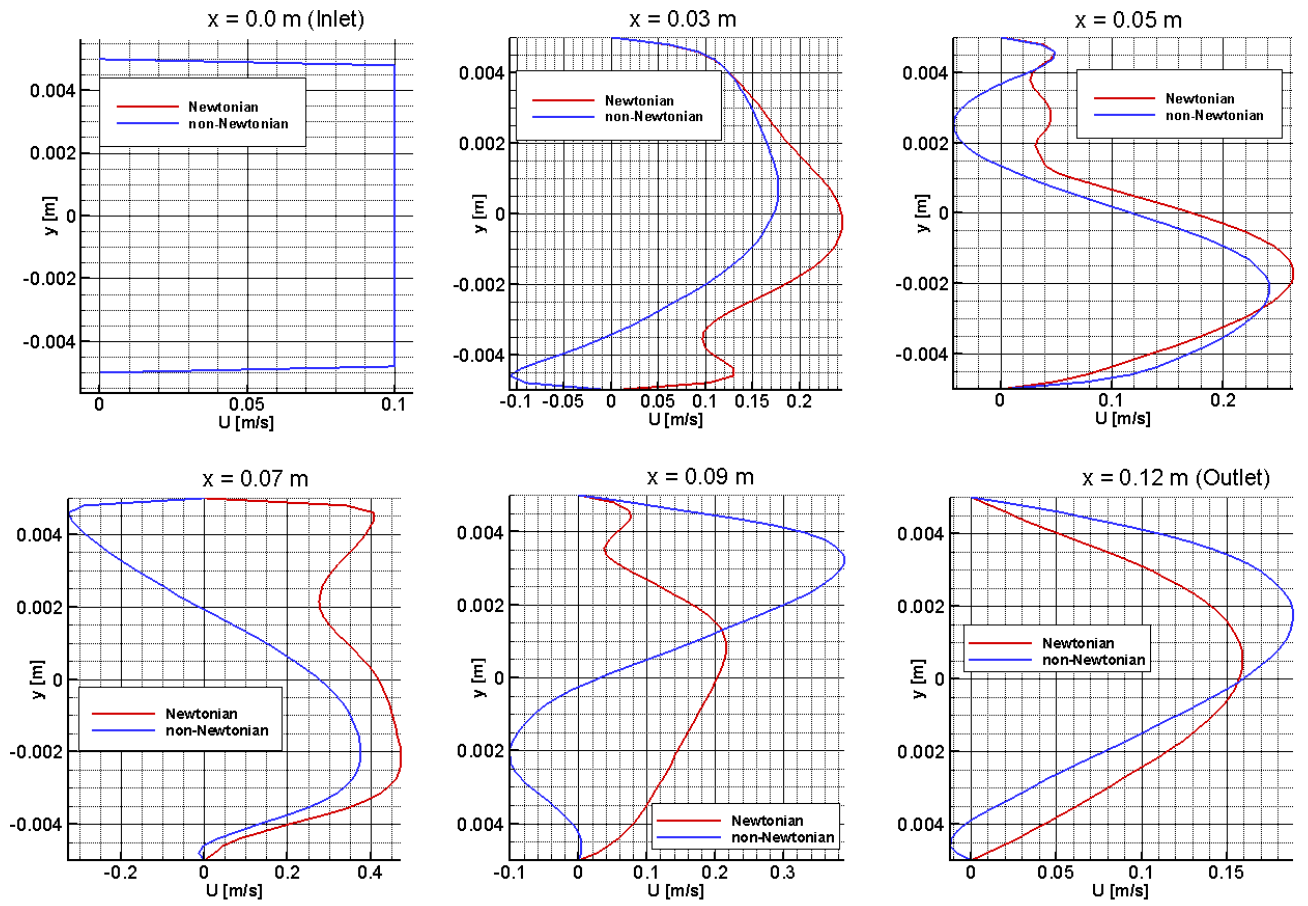

Figure 16: Comparison of velocity profiles for a non-isothermal blood flow in a microfluidic channel at different cross-sections when the applied magnetic field is induced by wires located on the lower and upper walls.

For an incompressible, laminar and non-isothermal MHD-FHD flow influenced by an applied double wire magnetic field for Newtonian and non-Newtonian fluids, the velocity and temperature profiles at different locations of the microfluidic channel have been shown in Figures 16 and 17, respectively. The reason for the numerical investigation presented in this subsection is that the double wire magnetic field arrangement has to trigger off a counter-clockwise and another clockwise rotating vortex at the location of the wires which leads to a similar vortical flow pattern to the benchmark problem of Tzirtzilakis and Loukopoulos [1] even if the fluid has to flow through a non-uniform applied magnetic field. The velocity and temperature fields are strongly affected at the locations of the applied magnetic field along with the flow upstream and downstream as well. A strong circulation regime can be observed closed to the magnetic field sources and the perturbation of the velocity field is significant about the wire located on the upper wall of the channel. When the fluid moves upstream, the flow influenced the fluid motion close to the wire located on the lower wall. The fluid flow downstream exhibits strong mixing due to the effect of the wire located on the upper wall. The difference between the Newtonian and non-Newtonian blood rheology approaches can be seen in Figures 16 and 17. 
In both cases, the velocity profile does not become fully-developed at the outlet section of the channel which is also valid for the temperature field. It also means that the flow supposed to be become fully-develop far downstream from the second wire located on the upper wall which could occur in a longer microfluidic channel. The difference of velocity and temperature distributions between Newtonian and non-Newtonian fluids becomes significant at the downsteam flow regime and at the outlet section of the channel. This phenomenon can be explained by supposing that the non-linear viscous effect of non-Newtonian blood flows turns out to be dominant when the magnetic field intensity is increased which comes from the material behaviour of blood. The difference could also arise purely due to the unstable numerical behaviour of the non-Newtonian blood flow in the presence of a strong magnetic field, because the numerical solution was not converging to a small threshold value for the steady-state non-Newtonian fluid flow in this case, thus the residual history appears to be strongly oscillatory (see Figure 18).
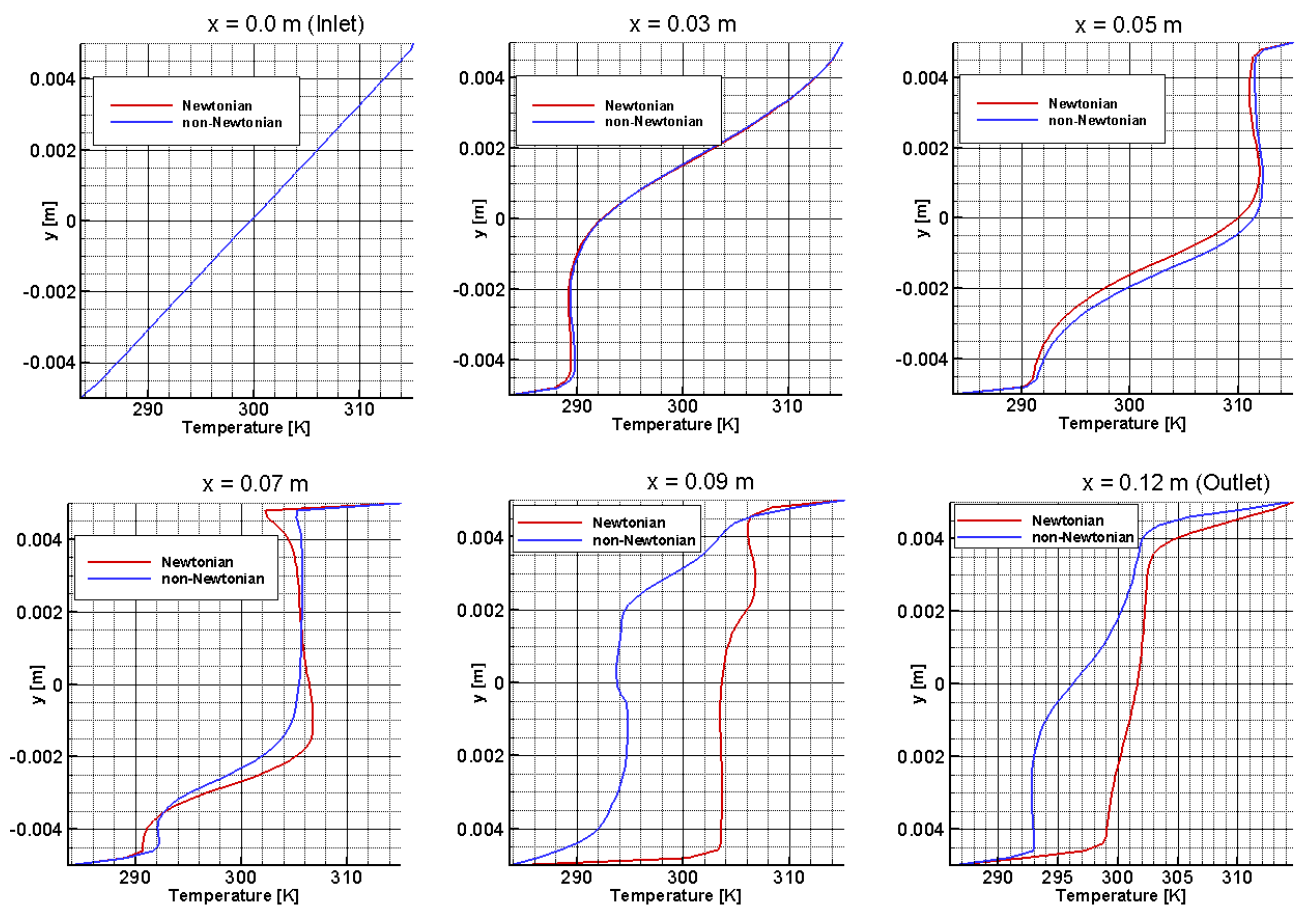

Figure 17: Comparison of temperature profiles for a non-isothermal blood flow in a microfluidic channel at different cross-sections when the applied magnetic field is induced by wires located on the lower and upper walls.

For the investigated steady-state Newtonian fluid flow in a microfluidic channel, the numerical solution of continuity and momentum equations was converging to a small threshold value of $10^{-14}$ while the solution of the temperature equation was converging up to the threshold value of $10^{-4}$ by performing 4000 total number of iterations. For non-Newtonian blood flow, the numerical solution exhibited strongly oscillatory behaviour (see Figure 18). Furthermore, this benchmark problem required the highest total number of iterations compared to the previous two numerical examples presented in this paper. It can also be seen relying on the residual histories (see Figure 18) that this benchmark could be used for MHD-FHD code verification and model validation for Newtonian fluids, however it might be considered as a non-conclusive example for non-Newtonian blood flows when the power-law fluid model is employed.

Relying on the obtained results, it can be seen that the simulation of non-isothermal MHD- 

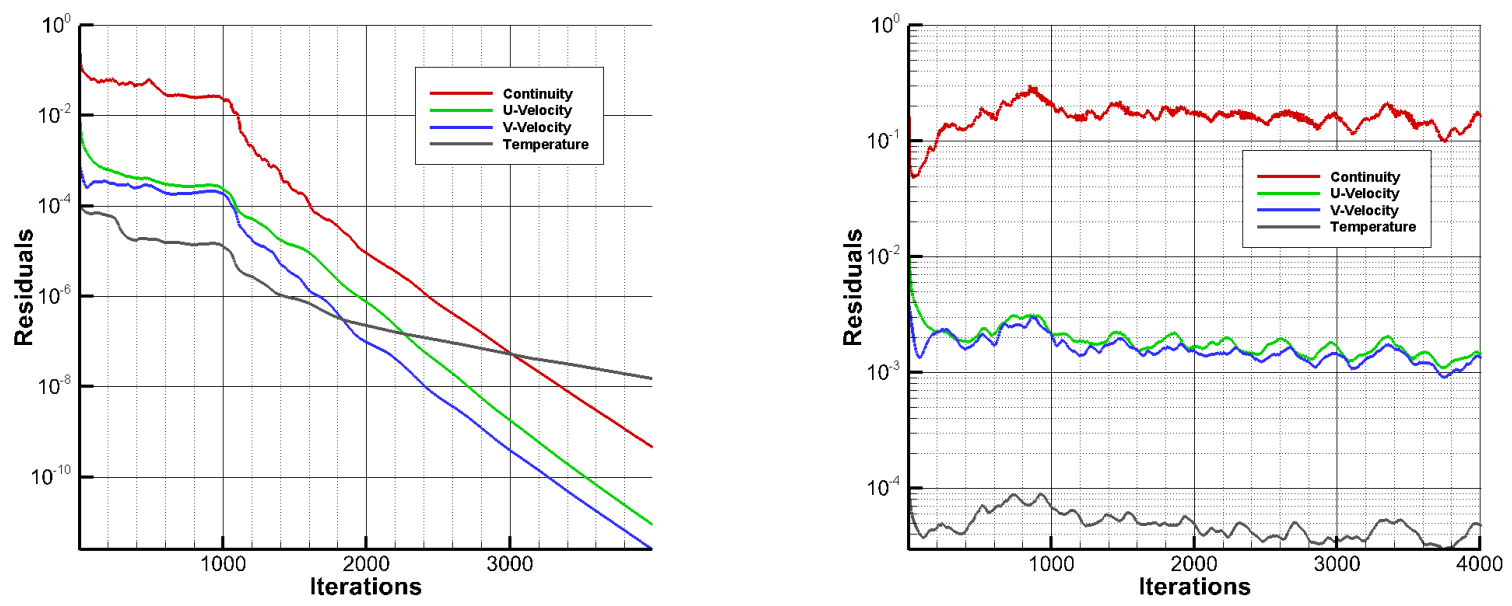

Figure 18: Residual history of non-isothermal blood flow simulations for a double wire problem: Newtonian fluid flow (left) and non-Newtonian blood flow (right).

FHD flows in conjunction with Newtonian and non-Newtonain blood rheological approaches for microfluidic and biomedical applications could be challenging. This is due to the fact that the stiff MHD and FHD source terms in the governing equations including the non-linear viscous behaviour of non-Newtonain blood flows could lead to oscillatory numerical solutions when the applied magnetic field strength is a high value. Therefore the behaviour of other numerical solution methods in conjunction with non-isothermal non-Newtonian blood flows has to be further investigated which could be a subject of another study.

\section{CONCLUSIONS}

In this paper, we proposed an incompressible, laminar and non-isothermal MHD-FHD model for Newtonian and non-Newtonian rheological models of blood relying on the combination of existing MHD-FHD approaches of Tzirakis et al. [2] and Tzirtzilakis [7]. This paper focused on the implementation of the proposed MHD and FHD models in the ANSYS-FLUENT environment for a) non-isothermal Newtonian and non-Newtonian Hartmann flows in the presence of an applied constant magnetic field and b) non-isothermal non-Newtonian blood flows relying on the power-law fluid model when the flow field was influenced by a single and double wire arrangement of an applied magnetic field in a microfluidic planar channel. The objective of this work was to provide reliable computational benchmark problems for CFD code verification and MHD-FHD model validation purposes. The following conclusions can be drawn as

- For solving an incompressible steady-state non-isothermal Hartmann flow problem in conjunction with Newtonian and non-Newtonian blood rheological models, the numerical solution of the MHD governing equations was converging to a small threshold value of $10^{-8}$ within 145 number of iterations. The obtained results have been compared to the computational data of Tzirakis et al. [2] taken from the literature. This benchmark problem can be considered as a reliable benchmark test case for code verification and MHD model validation purposes for non-isothermal non-Newtonian blood flows.

- When a single wire induced magnetic field was located on the lower wall of the microflu- 
idic channel for solving an incompressible steady-state non-isothermal MHD-FHD flow problem in conjunction with Newtonian and non-Newtonian blood rheological models, the numerical solution of the MHD-FHD governing equations was converging to a small threshold value of $10^{-7}$ within 450 total number of iterations. This validation test case can also be considered as a reliable computational benchmark to investigate the interaction between the temperature and MHD-FHD fields for non-Newtonian blood flows, because there is a lack of experimental data available in the literature.

- When a double wire arrangement of the applied magnetic field was located on the lower and upper walls of the microfluidic channel for solving an incompressible steady-state non-isothermal MHD-FHD flow problem related to Newtonian and non-Newtonian blood rheological models, the numerical solution of the MHD-FHD governing equations exhibited strongly oscillatory behaviour for non-Newtonian blood flows. Relying on these results, this benchmark case could only be used for MHD-FHD code verification and model validation for Newtonian fluids, however, it might be considered as a non-conclusive example for non-Newtonian blood flows when the power-law fluid model is employed.

- For solving incompressible non-isothermal MHD-FHD problems, it has been observed that the limitation of the current implementation is that when the applied magnetic field strength was higher than 4 Tesla, it was difficult to ensure convergence of the numerical solution for both Newtonian and non-Newtonian fluids. This limitation can be explained by the fact that the MHD-FHD source terms are stiff source terms in the momentum and energy equations, therefore the numerical behaviour of higher-order methods [11] should be further investigated for MHD-FHD problems at high dimensionless magnetic numbers.

\section{REFERENCES}

[1] Tzirtzilakis, E. E., Loukopoulos, V. C., Biofluid flow in a channel under the action of a uniform localized magnetic field. Computational Mechanics, Springer, 36(5), 360-374, 2005 .

[2] Tzirakis, K., Papaharilaou, Y., Giordano, D., Ekaterinaris, J., Numerical investigation of biomagnetic fluids in circular ducts. International Journal for Numerical Methods in Biomedical Engineering, 30(3), 297-317, 2014.

[3] Tzirakis, K., Botti, L., Giordano, D., Papaharilaou, Y., Numerical modelling of non-Newtonian polarizable and magnetizable fluid flow. 8th European Symposium on Aerothermodynamics for Space Vehicles, 2-6 March 2015, IST Congress Centre, Lisbon, Portugal, p. 152 (Abstract), Full Paper Published Online (http://www.congrexprojects.com), 2015.

[4] Tzirtzilakis, E. E., Sakalis, V. D., Kafoussias, N. G., Hatzikonstantinou, P. M., Biomagnetic fluid flow in a 3D rectangular duct, International Journal for Numerical Methods in Fluids, 44(12), 1279-1298, 2004.

[5] Loukopoulos, V. C., Tzirtzilakis, E. E., Biomagnetic channel flow in spatially varying magnetic field. International Journal of Engineering Science, 42, 571-590, 2004. 
[6] Könözsy, L., Multiphysics CFD Modelling of Incompressible Flows at Low and Moderate Reynolds Numbers, Ph.D. Thesis, Centre for Fluid Mechanics and Computational Science, Cranfield University, Cranfield, Bedfordshire, MK43 0AL, UK, 2012.

[7] Tzirtzilakis, E. E., A mathematical model for blood flow in magnetic field. Physics of Fluids, 17, 077103, 2005.

[8] Neofytou, P., Drikakis, D., Non-Newtonian flow instability in a channel with a sudden expansion. Journal of Non-Newtonian Fluid Mechanics, 111, 127-150, 2003.

[9] Scienza, P., Numerical Investigation of Non-Newtonian Biomagnetic Fluid Flows in Presence of Localized Magnetic Field, M.Sc. Thesis, Centre for Fluid Mechanics and Computational Science, Cranfield University, Cranfield, Bedfordshire, MK43 0AL, UK, 2015.

[10] ANSYS Inc., ANSYS FLUENT Theory Guide. ANSYS, Release 14.0, November, 2011.

[11] Könözsy, L., Drikakis, D., A Unified Fractional-Step, Artificial Compressibility and Pressure-Projection Formulation for Solving the Incompressible Navier-Stokes Equations. Communications in Computational Physics, 16(5), 1135-1180, 2014. 


\section{Validation of a magneto- and ferro-hydrodynamic model for} non-isothermal flows in conjunction with Newtonian and non-Newtonian fluids

Könözsy, László Z.

European Community on Computational Methods in Applied Sciences

L. Konozsy, P. Scienza, and D. Drikakis. Validation of a magneto- and ferro-hydrodynamic model for non-isothermal flows in conjunction with Newtonian and non-Newtonian fluids. Eccomas 2016 Proceedings ECCOMAS Congress 2016 VII European Congress on Computational Methods in Applied Sciences and Engineering, 5-10 June 2016, Creta Maris, Crete, Greece.

https://dspace.lib.cranfield.ac.uk/handle/1826/10478

Downloaded from Cranfield Library Services E-Repository 